\title{
First seizure presentation in an elderly woman with primary vitamin D deficiency: a case report
}

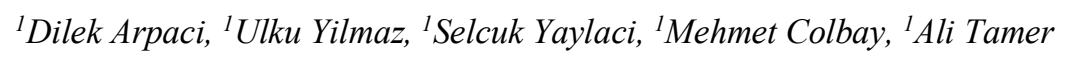

${ }^{\text {I} S a k a r y a}$ Education and Research Hospital,Internal medicine Department, Sakarya Turkey

\begin{abstract}
Introduction: Vitamin D insufficiency is common in older people and is associated with several disorders related to aging such as osteoporosis, which leads to a significantly increased risk of bone fractures. This deficiency is more common in Mediterranean countries than in Northern European countries. Hypocalcemic seizures resulting from vitamin D deficiency are rare in adults, and fractures caused by seizures without evidence of direct trauma have not yet been reported.
\end{abstract}

Case presentation: A 63-year-old Turkish woman was brought to the emergency department after having a first seizure,right forearm fracture (Figure 1) without trauma. Her vital signs were normal. Chvostek's and Trousseau's signs were positive. Other physical examination was normal. Brain computerized tomography $(\mathrm{CT})$ and brain magnetic resonance imaging (MRI) were normal. Her medical history included subtotal thyroidectomy. Her calcium level of 5.8 $\mathrm{mg} / \mathrm{dL}$ and phosporus level of $2.8 \mathrm{mg} / \mathrm{dL}$, albumin level was $3.8 \mathrm{~g} / \mathrm{dL}(3.5-5.2 \mathrm{~g} / \mathrm{dL})$. PTH level was $224 \mathrm{pg} / \mathrm{ml}, 25(\mathrm{OH})$ vitamin D level was $2.5 \mathrm{ng} / \mathrm{mL}$,alkaline phosphatase (ALP) level was $189 \mathrm{U} / \mathrm{L}$, bone alkaline phosphatase level was high.Urinary calcium excretion was low. Blood urine nitrogen (BUN), creatinine, sodium, potassium and magnesium levels were normal.An electrocardiogram showed a normal sinus rhythm with a QTc of 405 milliseconds. The patient received intravenous calcium gluconate. The patient was diagnosed with primary vitamin D deficiency. Vitamin D3 drops 50000 IU/week were given to the patient during 8 weeks; calcium carbonate/vitamin D3 effervescent tablets were also administered. A bone mineral density (BMD) scan was osteoporosis. Bisphosphonate treatment was postponed because of severe osteomalacia One month after starting vitamin D supplementation, serum 25-hydroxyvitamin D increased to a level of $28 \mathrm{ng} / \mathrm{mL}$ and PTH decreased to a level of $119 \mathrm{pg} / \mathrm{mL}$. Serum calcium was measured at $8.5 \mathrm{mg} / \mathrm{dL}$ and phosporus at $4.2 \mathrm{mg} / \mathrm{dL}$, within normal ranges. During hospitalization no seizures were observed

Conclusion: It is important to check for calcium levels in older patients who present with nonfebrile seizures.

Key words: Hypocalcemic seizure; vitamin D deficiency; elderly patient; bone fracture 


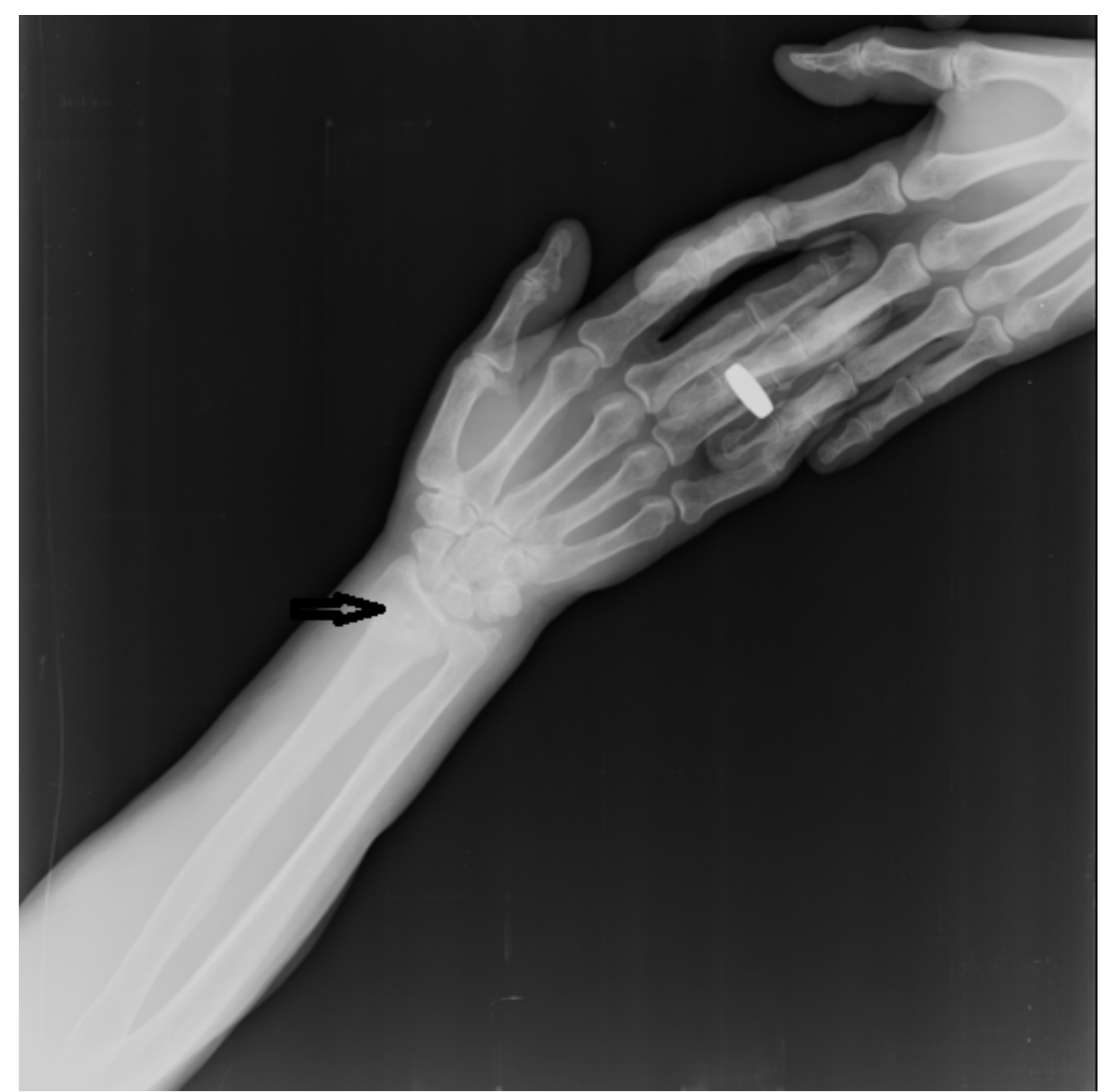

Figure 1. Right radius fracture was shown on X-ray (black arrow) 